\title{
PNPLA3 rs1010023 Predisposes Chronic Hepatitis B to Hepatic Steatosis but Improves Insulin Resistance and Glucose Metabolism
}

\author{
Qin Pan, ${ }^{1}$ Mei-Mei Chen, ${ }^{1}$ Rui-Nan Zhang, ${ }^{1}$ Yu-Qin Wang, ${ }^{1}$ Rui-Dan Zheng, ${ }^{2}$ Yu-Qiang Mi, ${ }^{3}$ \\ Wen-Bin Liu, ${ }^{4}$ Feng Shen, ${ }^{1}$ Qing Su, ${ }^{5}$ and Jian-Gao Fan ${ }^{1,6}$ \\ ${ }^{1}$ Department of Gastroenterology, Xinhua Hospital, Shanghai Jiaotong University School of Medicine, Shanghai 200092, China \\ ${ }^{2}$ Diagnosis and Treatment Center for Liver Diseases, Zhengxing Hospital, Zhangzhou, Fujian Province 363000, China \\ ${ }^{3}$ Department of Infectious Diseases, Tianjin Infectious Disease Hospital, Tianjin 300192, China \\ ${ }^{4}$ Wu-Jiao-Chang Community Health Center, Shanghai 200433, China \\ ${ }^{5}$ Department of Endocrinology, Xinhua Hospital, Shanghai Jiaotong University School of Medicine, Shanghai 200092, China \\ ${ }^{6}$ Shanghai Key Laboratory of Children's Digestion and Nutrition, Shanghai 200092, China
}

Correspondence should be addressed to Jian-Gao Fan; fattyliver2004@126.com

Received 28 December 2016; Revised 7 May 2017; Accepted 16 May 2017; Published 14 June 2017

Academic Editor: Ruozhi Zhao

Copyright @ 2017 Qin Pan et al. This is an open access article distributed under the Creative Commons Attribution License, which permits unrestricted use, distribution, and reproduction in any medium, provided the original work is properly cited.

\begin{abstract}
PNPLA3 polymorphisms serve as the genetic basis of hepatic steatosis in normal population and lead to dysregulated glucose metabolism. Whether it underlies the hepatic steatosis and glucose homeostasis in chronic hepatitis B patients remains uncertain. Here, we investigated the PNPLA3 polymorphisms in biopsy-proven chronic hepatitis B patients with (CHB+HS group, $n=52$ ) or without hepatic steatosis (CHB group, $n=47$ ) and non-CHB subjects with (HS group, $n=37$ ) or without hepatic steatosis (normal group, $n=45$ ). When compared to the TT genotype, C-allele at PNPLA3 rs1010023 (CC and TC genotypes) conferred higher risk to hepatic steatosis in chronic hepatitis B patients (odds ratio $(\mathrm{OR})=1.768,95 \%$ confidence interval $(\mathrm{CI}): 1.027-3.105 ; P=0.045)$ independent of age, gender, and body mass index. In contrast to their role in hepatic steatosis, CC and TC genotypes of PNPLA3 rs1010023 were correlated to significant improvement of homeostasis model assessment index (HOMA-IR) as compared to TT genotype in the CHB+HS group. Downregulated fasting blood glucose also characterized the $\mathrm{CHB}+\mathrm{HS}$ patients with C-allele at PNPLA3 rs1010023 (CC/TC versus TT: $4.81 \pm 0.92 \mathrm{mmol} / \mathrm{L}$ versus 5.86 $\pm 2.11 \mathrm{mmol} / \mathrm{L}, P=0.02$ ). These findings suggest that $P N P L A 3 \mathrm{rs} 1010023$ may predispose chronic hepatitis $\mathrm{B}$ patients to hepatic steatosis but protects them from glucose dysregulation by attenuating insulin resistance.
\end{abstract}

\section{Introduction}

By the high prevalence $(7.2 \%, 2006)$ of $\mathrm{HBV}$ infection, chronic hepatitis $\mathrm{B}(\mathrm{CHB})$ used to serve as the leading causes of chronic liver diseases (CLDs) in the Chinese population $[1,2]$. In contrast, the growing incidence of obesity and metabolic syndrome (MetS), mainly on the basis of western diets and unhealthy lifestyle, leads to a dramatic alternation in the spectrum of CLDs [2]. Hepatic steatosis, with the prevalence of $60-90 \%$ in obese patients, has recently replaced chronic hepatitis B to dominate the CLD in China [2]. As a result, hepatic steatosis occurs on the basis of chronic hepatitis B with an increasing annual prevalence from $8.2 \%$ (2002) to $13.5 \%-31.8 \%(2011)[3,4]$.

In contrast to simple chronic hepatitis $\mathrm{B}$, concurrence of chronic hepatitis B and hepatic steatosis demonstrates a significant impact on both insulin sensitivity and glucose metabolism [5-10]. By multivariate analysis, body mass index (BMI) [5-7, 9], fasting insulin [5], homeostasis model assessment index (HOMA-IR) [8-10], and fasting blood glucose (FBG) [6-8] are positively associated with the hepatic steatosis in different ethnicities independent of HBV infection. Glycosylated haemoglobin (HbAlc), another critical biomarker of glucose regulation, is correlated to 
indexes of hepatic steatosis, including ultrasonography scores (FLUS) and serum cholinesterase (ChE) [11]. On the other hand, hepatic steatosis exerts a prominent effect on both viral dynamics $[12,13]$ and sustained response to antiviral therapy [14, 15]. Host metabolic abnormality rather than viral factor is verified to be responsible for these effects $[6-7,16]$.

Single-nucleotide polymorphisms (SNPs) in patatinlike phospholipase domain-containing protein 3 (PNPLA3), which encodes adiponutrin in hepatocytes, has recently been proposed to confer the genetic susceptibility of hepatic steatosis. PNPLA3 rs738409 $\mathrm{C}>\mathrm{G}$ among these ones induces adiponutrin variant of $1148 \mathrm{M}$, an isoleucine-to-methionine substitution with reduced activity of triglyceride (TG) hydrolysis, and predisposes normal populations (Chinese, Japanese, Korean, Filipino, Indian, Turk, Belgian, Mexican, American, and so forth) to hepatic steatosis [6, 17-22]. Except for rs738409, some other PNPLA3 SNPs (rs2281135, rs139051, and rs2294918) also relate to increased risk of hepatic steatosis in ethnic groups of African, Caucasian, East Asian, and Mexican Americans [23, 24]. However, the role of PNPLA3 SNPs in patients with concurrent chronic hepatitis $\mathrm{B}$ and hepatic steatosis has not been well explored. Actions of PNPLA3 SNPs in BMI, FBG, and insulin resistance (IR) remain controversial until now [24-28].

We, therefore, investigated the PNPLA3 polymorphisms by deep sequencing in biopsy-proven chronic hepatitis B patients, with or without hepatic steatosis, from Southern, Central, and Northern China. The interaction between PNPLA3 SNPs and hepatic steatosis was determined by liver pathology. Biochemical characteristics of FBG, HbAlc, and HOMA-IR were further employed to highlight the metabolic effect of PNPLA3 SNPs in opinion of IR and glucose metabolism.

\section{Materials and Methods}

2.1. Study Populations. Forty-five normal controls (normal group), 47 patients with only biopsy-proven chronic hepatitis B (CHB group), 37 patients with hepatic steatosis (HS group), and 52 patients with biopsy-proven chronic hepatitis B and hepatic steatosis ( $\mathrm{CHB}+\mathrm{HS}$ group) were enrolled between January 2012 and June 2013. Subjects of normal, $\mathrm{CHB}$, and $\mathrm{CHB}+\mathrm{HS}$ groups were recruited from Zhengxing Hospital (Zhang Zhou, Southern China, $n=28$ ), Xinhua Hospital (Shanghai, Central China, $n=67$ ), and Tianjin Hospital of Infectious Diseases (Tianjin, Northern China, $n=49$ ). Patients of HS group were recruited from Xinhua Hospital. Participants with the following were excluded: type 2 diabetes, high alcohol intake $(>30 \mathrm{~g} / \mathrm{d}$ for men and $>20 \mathrm{~g} / \mathrm{d}$ for women), chronic HCV infection, autoimmune hepatitis, Wilson's disease, hereditary hemochromatosis, and hepatic steatosis related to current or previous treatment. The study was approved by the Ethics Committee of Xinhua Hospital. Informed consent was obtained from all subjects. Clinical investigations were conducted in appliance with the principles of Helsinki Declaration (1964).
2.2. Demographic, Anthropometric, and Biochemical Analysis. Demographic (age, gender) and anthropometric information (height, weight, BMI, hipline, and waistline) were characterized for the study population. Blood sample was collected from each patient and control subject after a 12-hour fasting. Biochemical tests were performed for measuring the activity of alanine aminotransferase (ALT), aspartate aminotransferase (AST), alkaline phosphatase (ALP), and gamma-glutamyltransferase ( $\gamma$-GT) activity, as well as the level of uric acid (UA), total bilirubin (TBIL), FBG, HbAlc, total cholesterol (TC), TG, high-density lipoprotein (HDL), and low-density lipoprotein (LDL) using multichannel automatic analyzer (Hitachi 7600, Tokyo, Japan).

2.3. Assessment of Insulin Sensitivity. Serum samples of different groups were harvested as mentioned above. The level of fasting insulin was quantified by ARCHITECT ${ }^{\circledR}$ insulin assay (Abbott Laboratories, Abbott Park, IL, United States) on ARCHITECT i2000 fully automated immunoassay analyzer (Abbott Laboratories, Abbott Park, IL, United States). HOMA-IR was used to evaluate insulin resistance [29].

HOMA-IR $=$ fasting serum insulin $(\mu \mathrm{IU} / \mathrm{ml}) \times$ fasting plasma glucose $(\mathrm{mmol} / \mathrm{L}) / 22.5$.

2.4. Hepatic Pathologic Analysis. Liver samples of patients were collected by needle biopsy after informed consent. Obtained liver tissues were then fixed in $10 \%$ buffered formalin, embedded in paraffin, and sliced for hematoxylin-eosin (H\&E) evaluation. Hepatic steatosis was graded from 0 to 3 based on the severity of steatosis at histological examination: S0: $<5 \%$, S1: 5-33\%, S2: 34-66\%, and S3: >66\% [30].

2.5. Genotyping of PNPLA3 SNPs. Blood samples obtained from the subjects were centrifuged at $1500 \mathrm{rpm}$ for $10 \mathrm{~min}$ immediately after sample collection. The buffy-coat layer was separated and transferred into $1.5 \mathrm{~mL}$ centrifuge tubes. Genomic DNA was successively extracted from the concentrated lymphocytes of the buffy coat using QIAamp DNA Mini Kit (Qiagen, Benlo, Limburg, Netherlands). Thereafter, the custom Ion AmpliSeq panel (Life Technologies of Thermo Fisher Scientific, Waltham, MA, United States) of PNPLA3 was designed, with the overall coverage rate of 89.91\%. Emulation PCR of the template was performed using the Ion OneTouch 2 System (Life Technologies of Thermo Fisher Scientific, Waltham, MA, United States) according to the manufacturer's instructions. PNPLA3 variants were genotyped by DNA sequencing using the Ion 318 Chip (Life Technologies of Thermo Fisher Scientific, Waltham, MA, United States) following the Ion PGM 200 Sequencing kit protocol.

2.6. Association Analysis for PNPLA3 SNPs and Clinical Phenotypes. The association test of PNPLA3 SNPs (rs1010023, rs738409), demographic (age, sex), anthropometric (height, weight, hipline, and waistline), and biochemical parameters (TBIL, DBIL, AST, ALT, GGT, ALP, INS, FBG, TC, TG, HDL, LDL, UA, and BUN), was carried out by logistic regression using PLINK v1.07 [31, 32]. 
TABle 1: Demographic, anthropometric, and clinical data.

\begin{tabular}{|c|c|c|c|c|c|}
\hline & Normal group & HS group & CHB group & $\mathrm{CHB}+\mathrm{HS}$ group & $P$ value \\
\hline Age (years) & $46.05 \pm 6.64$ & $38.98 \pm 13.55$ & $36.46 \pm 11.93$ & $39.82 \pm 13.89$ & $<0.001$ \\
\hline \multirow{2}{*}{ Gender } & M: 27 (60.00\%) & M: 27 (72.97\%) & M: 32 (68.09\%) & M: 38 (73.08\%) & \multirow{2}{*}{0.388} \\
\hline & F: $18(40.00 \%)$ & F: $10(27.03 \%)$ & F: 15 (31.91\%) & F: $14(26.92 \%)$ & \\
\hline BMI $\left(\mathrm{kg} / \mathrm{m}^{2}\right)$ & $23.12 \pm 2.18$ & $27.23 \pm 3.84$ & $22.59 \pm 2.47$ & $27.57 \pm 3.35$ & $<0.001$ \\
\hline TC (mmol/L) & $4.26 \pm 0.75$ & $4.67 \pm 1.30$ & $4.46 \pm 0.86$ & $4.77 \pm 0.83$ & 0.021 \\
\hline TG (mmol/L) & $1.00 \pm 0.35$ & $1.52 \pm 0.61$ & $1.15 \pm 0.41$ & $1.73 \pm 1.29$ & $<0.001$ \\
\hline HDL (mmol/L) & $1.32 \pm 0.24$ & $1.18 \pm 0.37$ & $1.36 \pm 0.25$ & $1.19 \pm 0.32$ & 0.092 \\
\hline $\mathrm{LDL}(\mathrm{mmol} / \mathrm{L})$ & $2.32 \pm 0.38$ & $3.01 \pm 0.62$ & $2.19 \pm 0.57$ & $2.92 \pm 0.87$ & $<0.001$ \\
\hline ALT (U/L) & $13.91 \pm 4.00$ & $50.44 \pm 22.56$ & $73.85 \pm 52.38$ & $61.19 \pm 32.59$ & $<0.001$ \\
\hline AST (U/L) & $20.19 \pm 4.84$ & $27.24 \pm 14.47$ & $70.56 \pm 49.54$ & $33.74 \pm 20.51$ & $<0.001$ \\
\hline TBIL $(\mu \mathrm{mol} / \mathrm{L})$ & $2.22 \pm 0.42$ & $4.62 \pm 0.93$ & $21.48 \pm 14.14$ & $5.86 \pm 2.54$ & $<0.001$ \\
\hline GGT (U/L) & $15.05 \pm 4.94$ & $43.91 \pm 20.83$ & $73.79 \pm 64.96$ & $64.04 \pm 39.57$ & $<0.001$ \\
\hline ALP (U/L) & $16.31 \pm 4.61$ & $61.58 \pm 16.17$ & $93.66 \pm 35.80$ & $89.11 \pm 42.48$ & $<0.001$ \\
\hline Insulin $(\mu \mathrm{IU} / \mathrm{L})$ & $7.51 \pm 1.86$ & $34.37 \pm 23.61$ & $8.10 \pm 2.37$ & $30.95 \pm 23.16$ & $<0.001$ \\
\hline HOMA-IR & $1.11 \pm 0.42$ & $8.25 \pm 6.07$ & $1.33 \pm 0.42$ & $7.38 \pm 5.63$ & $<0.001$ \\
\hline FBG $(\mathrm{mmol} / \mathrm{L})$ & $3.57 \pm 1.13$ & $5.14 \pm 1.17$ & $4.15 \pm 0.45$ & $5.48 \pm 1.83$ & $<0.001$ \\
\hline Hb1Ac (\%) & $5.28 \pm 0.97$ & $5.82 \pm 1.29$ & $5.17 \pm 1.01$ & $6.12 \pm 1.43$ & $<0.001$ \\
\hline
\end{tabular}

CHB: chronic hepatitis B; HS: hepatic steatosis; BMI: body mass index; TC: total cholesterol; TG: triglyceride; HDL: high-density lipoprotein; LDL: low-density lipoprotein; FBG: fasting blood glucose; ALT: alanine aminotransferase; AST: aspartate aminotransferase; TBIL: total bilirubin; GGT: $\gamma$-glutamyltransferase ALP: alkaline phosphatase; HOMA-IR: homeostasis model assessment index.

2.7. Statistical Analysis. The data are expressed as mean \pm SD. Age- and gender-adjusted odds ratios (ORs) were calculated using multivariant logistic regression with genotypes, age, and gender as the independent variables. Chi-square test was used to test differences in genotype distribution. Differences among the groups of genotypes were tested by ANOVA using SPSS version 16.0 (SPSS Inc., Chicago, IL, United States). Differences were considered to be statistically significant at a $P$ value $<0.05$.

\section{Results}

3.1. Anthropometric and Clinical Data. Patients with hepatic steatosis (HS group, $\mathrm{CHB}+\mathrm{HS}$ group) exhibited BMI much higher than that of the chronic hepatitis $B$ patients and normal controls $(P<0.001$, Table 1$)$. When compared to those without hepatic steatosis (CHB group, normal group), patients of the HS and $\mathrm{CHB}+\mathrm{HS}$ group also suffered from the increased levels of TG, TC, LDL, and FBG (all $P<0.05$ ) and a trend for decreased HLD (Table 1). Impaired glucose homeostasis and lipid metabolism were then suggested in the $\mathrm{CHB}+\mathrm{HS}$ patients independent of chronic hepatitis $\mathrm{B}$. For the sake of coexisted chronic hepatitis B, which reflects the chronic hepatic inflammation, there was no significant difference in ALT and AST activities between the $\mathrm{CHB}+\mathrm{HS}$ and $\mathrm{CHB}$ groups.

In both $\mathrm{HS}$ and $\mathrm{CHB}+\mathrm{HS}$ groups, subjects with $\mathrm{C}$-allele at PNPLA3 rs1010023 (CC and TC genotypes) exhibited BMI much lower than those with T-allele (TT genotype) $(P<0.05$, Table 2$)$. Similarly, a decreasing tendency of BMI characterized the normal subjects with CC and TC genotypes, instead of TT genotype, of PNPLA3 rs1010023 (Table 2).
3.2. PNPLA3 rs1010023 Associated with Lipid Metabolism and Hepatic Steatosis in CHB Patients. In normal, HS, and $\mathrm{CHB}+\mathrm{HS}$ groups, subjects bearing $\mathrm{C}$-allele (CC and TC genotypes) at PNPLA3 rs1010023 demonstrated fasting TG level statistically lower than those with both T-alleles (TT genotype) (CC/TC versus TT: $0.86 \pm 0.22 \mathrm{mmol} / \mathrm{L}$ versus $1.06 \pm 0.37 \mathrm{mmol} / \mathrm{L}, P=0.03$ (normal group); $1.21 \pm$ $0.46 \mathrm{mmol} / \mathrm{L}$ versus $1.72 \pm 0.64 \mathrm{mmol} / \mathrm{L}, P=0.04$ (HS group); $1.22 \pm 0.41 \mathrm{mmol} / \mathrm{L}$ versus $1.96 \pm 1.48 \mathrm{mmol} / \mathrm{L}, \quad P=0.04$ (CHB+HS group)) (Table 2). Among these subjects, there was a slight reduction in the TC and LDL levels and a moderate increase in the HDL level (Table 2).

Genotyping for PNPLA3 polymorphisms, the C-allele at rs1010023 (CC and TC genotypes) was associated with hepatic steatosis in CHB patients (odds ratio $(\mathrm{OR})=1.78$, 95\% confidence interval (CI): 1.05-3.03; $P=0.03$ ) (Table 3). A significant association between hepatic steatosis and PNPLA3 rs1010023 was further confirmed after adjusting for age, gender, and BMI (OR $=1.77,95 \% \mathrm{CI}$ : 1.03-3.11; $P=0.045$ ) (Table 3). In addition, CC/TC at PNPLA3 rs1010023 proved their association with patients of the HS group (Table 3). In contrast to the statistical difference of PNPLA3 rs1010023 polymorphism among $\mathrm{CHB}+\mathrm{HS}$ and nonsteatosis groups, there was a similar percentage of C-allele at rs1010023 between the groups of normal and $\mathrm{CHB}$, regardless of age, gender, and BMI adjustment (Table 3).

To evaluate the relationship between PNPLA3 rs1010023 and pathological features, severity of hepatic steatosis was investigated in both $\mathrm{CHB}+\mathrm{HS}$ and $\mathrm{HS}$ groups. As compared to those with TT genotype at rs1010023, patients harboring CC and TC genotypes at rs1010023 showed no association with significant steatosis $(>$ S1) (Table 4). 


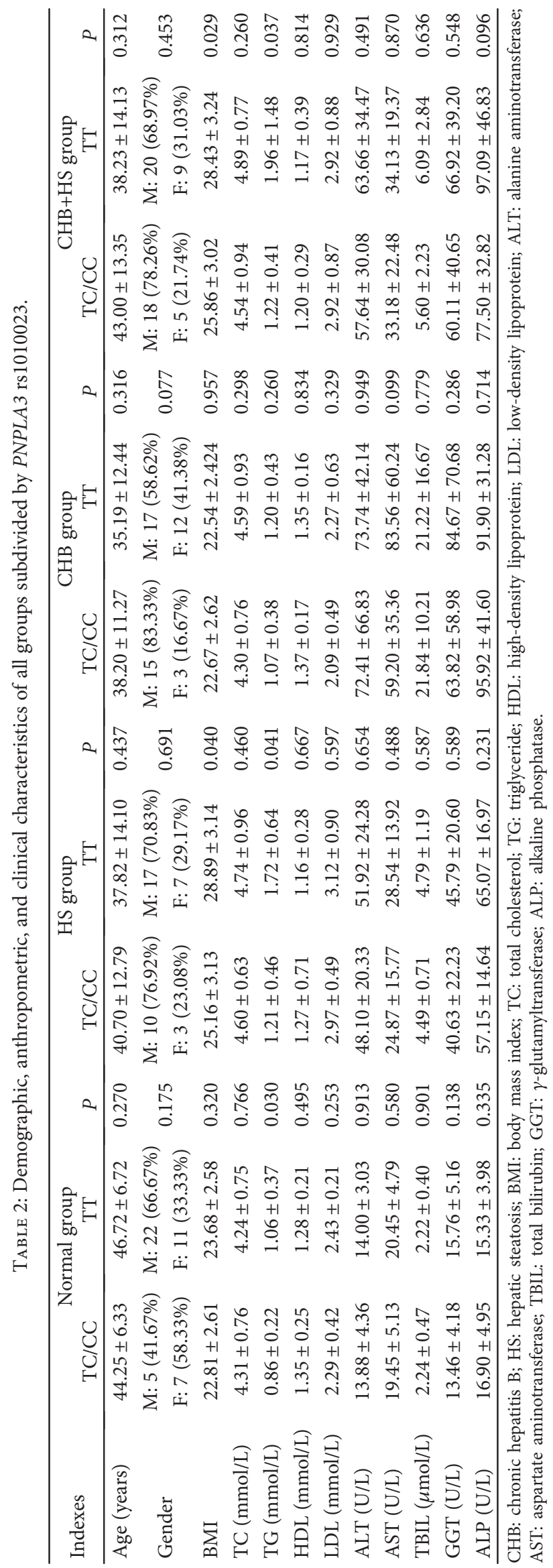




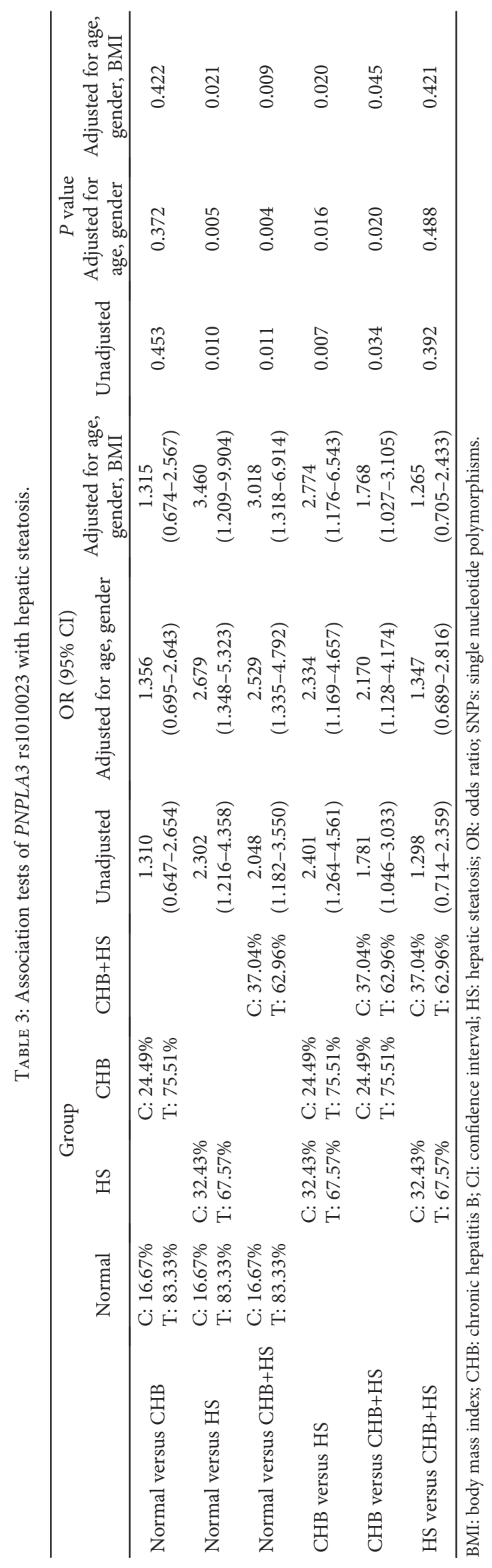


TABLE 4: Association tests of PNPLA3 rs1010023 with steatosis grade.

\begin{tabular}{lcccc}
\hline $\begin{array}{l}\text { Steatosis } \\
\text { grade }\end{array}$ & \multicolumn{2}{c}{ HS group } & \multicolumn{2}{c}{ CHB+HS group } \\
& TCC & TT & TC/CC & TT \\
\hline S1 & $4(30.77 \%)$ & $5(19.23 \%)$ & $8(36.36 \%)$ & $10(31.25 \%)$ \\
$>$ S1 & $9(69.23 \%)$ & $21(80.77 \%)$ & $14(63.64 \%)$ & $22(68.75 \%)$ \\
$P$ value & \multicolumn{2}{c}{0.420} & \multicolumn{2}{c}{0.695} \\
\hline
\end{tabular}

CHB: chronic hepatitis B; HS: hepatic steatosis.

3.3. PNPLA3 rs1010023 Increased Insulin Sensitivity. The normal, $\mathrm{CHB}, \mathrm{HS}$, and $\mathrm{CHB}+\mathrm{HS}$ groups were stratified by PNPLA3 genotypes and then subjected to comparisons on the basis of insulin sensitivity and $\beta$-cell function. When compared to those of the normal and $\mathrm{CHB}$ groups, patients of both $\mathrm{HS}$ and $\mathrm{CHB}+\mathrm{HS}$ group exhibited upregulated fasting insulin and HOMA-IR $(P<0.05)$ (Table 5).

Dramatically, C-allele (CC and TC genotypes) of PNPLA3 rs1010023 was correlated with HOMA-IR significantly lower than that of T-allele (TT genotype) in both $\mathrm{CHB}+\mathrm{HS}$ (CC/TC versus TT: $4.98 \pm 3.14$ versus $9.98 \pm 6.64, P=0.031$ ) and HS groups (CC/TC versus TT: $5.65 \pm 3.26$ versus $11.15 \pm 7.29, P=0.045$ ) (Table 5). Similar observations of serum insulin concentration and HOMA-IR, yet without statistical significance, could be obtained in nonsteatosis subjects (normal group, $\mathrm{CHB}$ group) with C-allele at rs1010023 (Table 5). Thus, phenotype of PNPLA3 rs1010023 may sensitize subjects to insulin and attenuate IR in patients with HS.

3.4. PNPLA3 rs1010023 Improved Glucose Homeostasis. Critical indexes (FBG, HbA1c) that related to glucose metabolism were evaluated in blood samples obtained from normal, $\mathrm{CHB}, \mathrm{HS}$, and $\mathrm{CHB}+\mathrm{HS}$ groups, respectively. As a result, obvious upregulation of FBG and $\mathrm{HbAlc}$ characterized the patients of $\mathrm{HS}$ and $\mathrm{CHB}+\mathrm{HS}$ groups (Table 1).

In both $\mathrm{HS}$ and $\mathrm{CHB}+\mathrm{HS}$ group, hepatic steatosis patients bearing $\mathrm{CC}$ and TC genotypes of PNPLA3 rs1010023 were susceptible to decreased level of FBG in comparison to those with TT genotype (CC/TC versus TT: $4.81 \pm 0.92 \mathrm{mmol} / \mathrm{L}$ versus $5.86 \pm 2.11 \mathrm{mmol} / \mathrm{L} \quad(\mathrm{CHB}+\mathrm{HS}$ group), $P=0.017 ; 4.27 \pm 0.82 \mathrm{mmol} / \mathrm{L}$ versus $5.52 \pm 1.11$ $\mathrm{mmol} / \mathrm{L}$ (HS group), $P=0.003$ ) (Table 5). There was also mild downregulation of FBG in the normal and $\mathrm{CHB}$ groups (Table 5).

Besides, decreasing tendency of $\mathrm{HbAlc}$, yet without statistical significance, characterized the subjects containing C-allele at rs1010023 in groups with or without hepatic steatosis (Table 5). Similar observations in both FBG and HbAlc suggested an improving effect of PNPLA3 rs101002 on glucose homeostasis, especially in chronic hepatitis B patients with hepatic steatosis.

3.5. PNPLA3 rs1010023 Shared Hepatosteatosis Susceptibility but Not Glucometabolic Effect with rs738409. In opinion to the risk of hepatic steatosis, an intimate association of PNPLA3 SNPs (rs1010023, rs738409) was revealed with statistical significance $\left(P=2.18 \times 10^{-26}\right)$ (Figure 1). Despite their similar effect on steatotic susceptibility, PNPLA3 rs738409 differed from rs1010023 in its glucometabolic characteristics. When compared to those with GG and GC genotypes, the CC genotype of PNPLA3 rs738409 conferred no risk to increased serum insulin and HOMA-IR in groups of $\mathrm{HS}$ and $\mathrm{CHB}+\mathrm{HS}$ (Table 6). Consistently, there was no statistical difference in both FBG and $\mathrm{HbAlc}$ between subjects carrying G- and C-allele at rs738409 (Table 6). PNPLA3 rs738409, therefore, does not exert significant impact on insulin sensitivity and glucose metabolism.

\section{Discussion}

Hepatic steatosis, an important component of metabolic syndrome, is now accepted to introduce the pathological disorders of nonalcoholic fatty liver disease (NAFLD) on the basis of "two-hit" mechanism [33]. Hepatocyte-specific lipid (mainly TG) accumulation reflects the "first hit," which is recently resulted from the western lifestyle with high-fat diet in the Chinese population [34]. Then, hepatic steatosis based on lipid accumulation predisposes subjects to the "second hit" of lipoperoxidation and oxidative stress [34]. Thus, hepatic steatosis serves as the initiation of NAFLD, which ranges from simple steatosis to nonalcoholic steatohepatitis (NASH) with clinical outcomes of liver fibrosis/cirrhosis and hepatocellular carcinoma (HCC) [2]. Physiologically, dietary TG is absorbed and transported to hepatocytes by circulating chylomicrons. Low concentration, yet in steady state, of hepatic TG can be diverted from the cytosolic storage pool in a form of serum very low-density lipoprotein (VLDL), and finally be uptaken by systemic adipose tissue [35]. In contrast, steatosis occurs upon the unbalance of TG metabolism, especially excessive acquisition (i.e., high-fat diet) and decreased disposal (fatty acid oxidation and secretion of TG-rich lipoproteins), in hepatoyctes [36]. Dysregulation of hepatic TG metabolism, therefore, is suggested to introduce hepatic steatosis.

PNPLA3, a single-pass type II membrane protein with patatin-like domain at the N-terminal, has been characterized to be the multifunctional enzyme with both triacylglycerol lipase and acylglycerol $\mathrm{O}$-acyltransferase activities in hepatocytes [37]. The effect of PNPLA3 on triacylglycerol hydrolysis qualifies itself for a pivotal regulator of TG metabolism in the liver [38]. In the present study, C-allele of a novel PNPLA3 polymorphism, rs1010023, was uncovered to significantly associate with the susceptibility to hepatic steatosis in chronic hepatitis B patients from Southern, Central, and Northern China. This action was further proved to be independent of age, gender, and BMI after statistical adjustment. In consistent with other steatosis-related SNPs (i.e., rs738409, rs2281135, rs139051, and rs2294918) [17-24], PNPLA3 rs1010023 seems to be loss-of-function in the aspect of TG hydrolysis. Because of its location on the surface of lipid droplets, PNPLA3 with decreased adiponutrin activity in subjects carrying C-allele at rs 1010023 is suggested to downregulate the TG lipolysis $[39,40]$, which successively inhibits the oxidation and mobilization of fatty acids from the liver to peripheral adipose tissues [41]. The accumulation of TG-rich lipid droplets resultantly induces hepatic steatosis with diagnostic criteria of over 5\% [30]. 


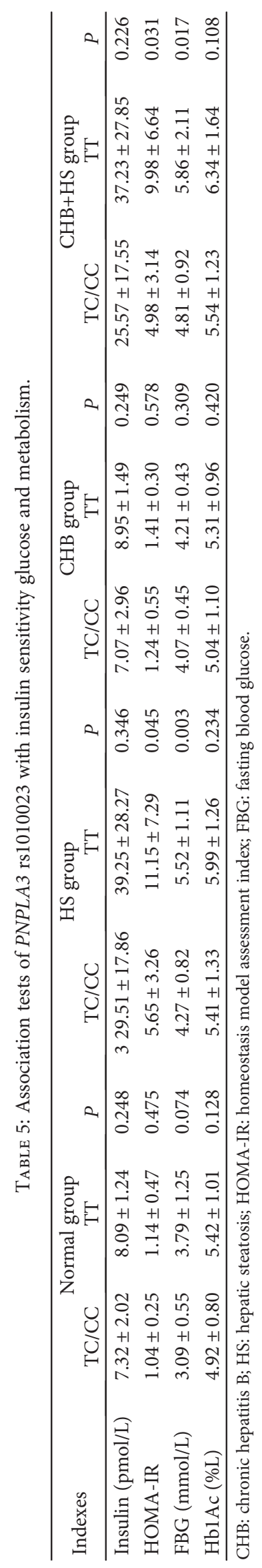




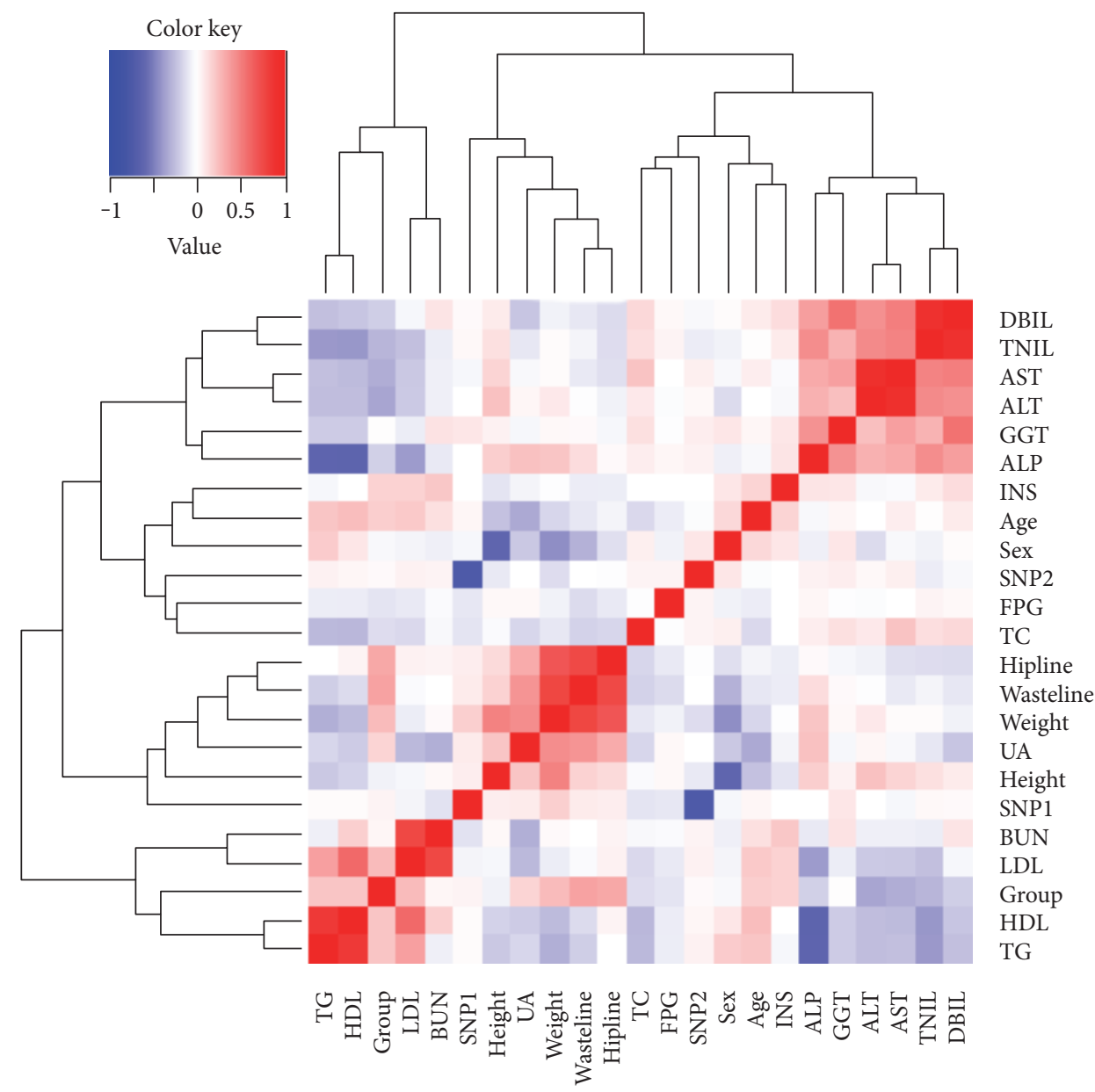

FIGURE 1: Association results are shown for the PNPLA3 SNPs and clinical phenotypes. ALT: alanine aminotransferase; ALP: alkaline phosphatase; AST: aspartate aminotransferase; BUN: blood urea nitrogen; DBIL: direct bilirubin; FBG: fasting blood glucose; GGT: gamma-glutamyltransferase; HDL: high-density lipoprotein; INS: insulin; LDL: low-density lipoprotein; SNP1: PNPLA3 rs738409; SNP2: PNPLA3 rs1010023; TBIL: total bilirubin; TC: total cholesterol; TG: triglyceride; UA: uric acid.

To take deep insight into the role of PNPLA3 rs1010023 within pathological progression, the degree of hepatocyte steatosis was assessed according to the SAF criteria. As a result, no significant association could be observed between PNPLA3 rs1010023 and the severity of hepatic steatosis (S1 or $>$ S1). Thus, PNPLA3 rs1010023 is indicated to underlie the occurrence of liver steatosis in chronic hepatitis $\mathrm{B}$ patients. Interestingly, the percentage of $\mathrm{C}$-allele at PNPLA3 rs1010023 was similar between the normal and chronic hepatitis B groups, regardless of age and gender adjustment, suggesting the host metabolism rather than viral infection to be responsible for hepatic steatosis in Chinese chronic hepatitis B patients.

It has been demonstrated that the intracellular TG of hepatocytes undergoes lipolysis, and follows by re-esterification so as to incorporate into VLDL particle within the endoplasmic reticulum [35]. Therefore, reduction of PNPLA3based lipolysis may minimize the VLDL formation, and subsequently the outward transport of hepatic TG. When compared to those with TT genotype, there was indeed a significant decrease of fasting TG level in subjects carrying
CC and TC genotypes at rs1010023, no matter in the groups of normal, HS, and $\mathrm{CHB}+\mathrm{HS}$. Similarly, decreased TG level characterizes the NAFLD patients with T-allele at PNPLA3 rs139051 [24]. These findings shed light on a paradoxical dissociation between hepatosteatosis susceptibility and improved serum TG on the basis of rs1010023, and perhaps other SNPs, of PNPLA3.

Resulting from its disturbing effect on TG metabolism, PNPLA3 rs1010023 plays an important role in the hepatic and peripheral lipid distribution. A theoretic scenario is proposed that C-allele-dependent enzymatic loss of PNPLA3 hampers the TG transportation from hepatocytic lipid droplets to adipose tissues, with clinical features of liver steatosis and lowered serum level of TG, and then protects subjects from progressive obesity $[35,36,39-41]$. As evaluated by BMI, both $\mathrm{HS}$ and $\mathrm{CHB}+\mathrm{HS}$ patients carrying $\mathrm{CC}$ and TC genotype of PNPLA3 rs1010023 showed much less sensitive to obesity in comparison to those with TT genotype.

Recent studies have verified the prominent impact of obesity ( $\mathrm{BM} \geq 71.3$ th-85th percentile) on IR in various age and ethnic groups [42-45]. When assessed at a tissue-specific 


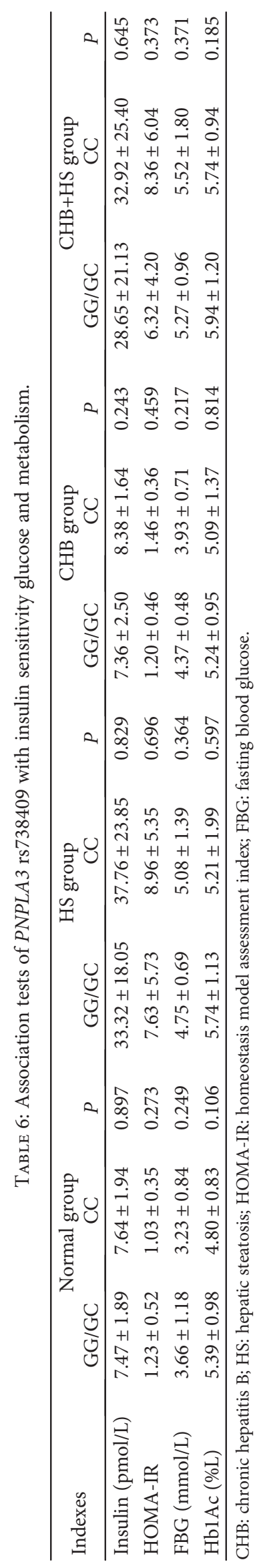


level, subcutaneous adipose tissue in the nonalcoholic steatohepatitis patients exhibits IR, with much insulin ( $>6$-fold) to cause less suppression of glycerol release (1/2-maxima level), even seriously than that of liver and skeleton muscle [46]. Intra-abdominal fat mass in polycystic ovary syndrome (PCOS) women shows a positive relation to the up-regulated serum level of fasting insulin, indicating the existence of IR [47]. Mechanically, excessive peripheral lipid, no matter the subcutaneous and intra-abdominal fat mass, promotes the release of free fatty acids (FFAs) [48]. Enlarged adipocytes are also integral to the increased secretion of proinflammatory chemokines and cytokines (i.e., MCP-1, TNF- $\alpha$, IL-1, IL-6, and IL-8) [49-51]. Both FFAs and obesityinduced inflammatory response serve as critical stimulators of systemic IR. As a result, IR specific to peripheral lipid facilitates the dysregulation of glucose metabolism [46, 47, 52]. Our experiments confirmed that rs1010023 Callele carriers in both HS and $\mathrm{CHB}+\mathrm{HS}$ groups were protected from IR and hyperglycemia, which featured the TT genotype carriers with significantly elevated levels of HOMA-IR and FBG. Thus, inverse correlation between peripheral lipid and insulin sensitivity may exhibit the mechanisms underlying the improvement of glycolipid metabolism [53]. Moreover, other risk SNPs (e.g., PNPLA3 rs139051) for NAFLD have recently been revealed to associate with reduced levels of BMI and IR [28, 54].

Except for the results obtained from normal and CHB + HS groups, another noticeable observation of the present study lied in that PNPLA3 rs1010023 did not associate with the levels of TG, insulin, FBG, and HOMA-IR in chronic hepatitis B patients. The protective role of HBV infection in glycolipid metabolism and related diseases, which are characterized by lower prevalence of NAFLD, hypertriglyceridemia, MetS [3, 6, 55], and IR rate [56], are likely to counteract the effect of PNPLA3 polymorphism. PNPLA3 rs738409, also known as I148M, has been well established to act as the genetic basis of NAFLD [17-28, 39, 41, 57]. With respect to the association of polymorphisms, PNPLA3 rs1010023 and rs738409 shared the susceptibility to hepatic steatosis in our experiments. But they differed from each other in aspects of obesity, IR, and glucose metabolism, respectively. In contrast to the obesity risk for T-allele at rs1010023, there was no statistical difference in BMI between subjects with C- and G-allele at rs738409 [57]. Furthermore, similar glycolipid indexes (TG, insulin, FBG, and $\mathrm{Hb} 1 \mathrm{Ac}$ ) and HOMA-IR characterized the patients with CC-, CG-, and GG-genotypes of rs738409 in both HS and CHB+HS groups [57]. Limited effect of PNPLA3 rs738409 on the redistribution of total fat mass could be responsible for these presentations.

Some limitations of the study should be considered. By reason of its biopsy-proven, steatosis-predisposing characteristics in parallel to that of other SNPs, PNPLA3 rs1010023 is supposed to function in a loss-of-function pattern. Nevertheless, mechanic study would highlight the precise role of PNPLA3 rs1010023 during TG metabolism. Second, comparison of rs1010023 and SNPs other than rs738409 may provide us with preferable understanding of PNPLA3 polymorphisms related to hepatic steatosis and glycolipid metabolism.

\section{Conclusions}

PNPLA3 rs1010023 predisposes chronic hepatitis B patients to hepatic steatosis in the Chinese Han population. Contrastively, PNPLA3 rs1010023 protects them from glucose dysregulation by attenuating the insulin resistance, probably on the basis of BMI reduction.

\section{Conflicts of Interest}

The authors declare that they have no conflicts of interest.

\section{Authors' Contributions}

Qin Pan, Mei-Mei Chen, and Rui-Nan Zhang contributed equally to this paper.

\section{Acknowledgments}

The work is supported by the State Key Development Program for Basic Research of China (no. 2012CB517501); National Natural Science Foundation of China (nos. 81070322, 81270491, and 81470840); 100 Talents Program (no. XBR2011007h); and Program of the Shanghai Committee of Science and Technology (nos. 09140903500 and 13ZR14267).

\section{References}

[1] Y. Cui and J. Jia, "Update on epidemiology of hepatitis B and C in China," Journal of Gastroenterology and Hepatology, vol. 28, Supplement 1, pp. 7-10, 2013.

[2] F. S. Wang, J. G. Fan, Z. Zhang, B. Gao, and H. Y. Wang, "The global burden of liver disease: the major impact of China," Hepatology, vol. 60, no. 6, pp. 2099-2108, 2014.

[3] V. W. Wong, G. L. Wong, W. C. Chu et al., "Hepatitis B virus infection and fatty liver in the general population," Journal of Hepatology, vol. 56, no. 3, pp. 533-540, 2012.

[4] M. M. Wang, G. S. Wang, F. Shen, G. Y. Chen, Q. Pan, and J. G. Fan, "Hepatic steatosis is highly prevalent in hepatitis B patients and negatively associated with virological factors," Digestive Diseases and Sciences, vol. 59, no. 10, pp. 25712579, 2014.

[5] A. L. Nau, J. C. Soares, M. B. Shiozawa, E. B. Dantas-Corrêa, L. Schiavon Lde, and J. L. Narciso-Schiavon, "Clinical and laboratory characteristics associated with dyslipidemia and liver steatosis in chronic HBV carriers," Revista da Sociedade Brasileira de Medicina Tropical, vol. 47, no. 2, pp. 158-164, 2014.

[6] Y. L. Cheng, Y. J. Wang, W. Y. Kao et al., "Inverse association between hepatitis B virus infection and fatty liver disease: a large-scale study in populations seeking for check-up," PloS One, vol. 8, no. 8, article e72049, 2013.

[7] R. D. Zheng, J. N. Chen, Q. Y. Zhuang, Y. H. Lu, J. Chen, and B. F. Chen, "Clinical and virological characteristics of chronic hepatitis B patients with hepatic steatosis," International Journal of Medical Sciences, vol. 10, no. 5, pp. 641-646, 2013.

[8] A. S. Dassanayake, A. Kasturiratne, S. Rajindrajith et al., "Prevalence and risk factors for non-alcoholic fatty liver disease among adults in an urban Sri Lankan population," 
Journal of Gastroenterology and Hepatology, vol. 24, no. 7, pp. 1284-1288, 2009.

[9] B. Yilmaz, S. Koklu, H. Buyukbayram et al., "Chronic hepatitis B associated with hepatic steatosis, insulin resistance, necroinflammation and fibrosis," African Health Sciences, vol. 15, no. 3, pp. 714-718, 2015.

[10] C. C. Wang, C. S. Hsu, C. J. Liu, J. H. Kao, and D. S. Chen, "Association of chronic hepatitis B virus infection with insulin resistance and hepatic steatosis," Journal of Gastroenterology and Hepatology, vol. 23, no. 5, pp. 779-782, 2008.

[11] S. Katoh, M. Peltonen, T. Wada et al., "Fatty liver and serum cholinesterase are independently correlated with HbA1c levels: cross-sectional analysis of 5384 people," The Journal of International Medical Research, vol. 42, no. 2, pp. 542-553, 2014.

[12] C. M. Chu, D. Y. Lin, and Y. F. Liaw, "Does increased body mass index with hepatic steatosis contribute to seroclearance of hepatitis B virus (HBV) surface antigen in chronic HBV infection," International Journal of Obesity, vol. 31, no. 5, pp. 871-875, 2007.

[13] M. V. Machado, A. G. Oliveira, and H. Cortez-Pinto, "Hepatic steatosis in hepatitis B virus infected patients: meta-analysis of risk factors and comparison with hepatitis C infected patients," Journal of Gastroenterology and Hepatology, vol. 26, no. 9, pp. 1361-1367, 2011.

[14] X. Jin, Y. P. Chen, Y. D. Yang, Y. M. Li, L. Zheng, and C. Q. Xu, "Association between hepatic steatosis and entecavir treatment failure in Chinese patients with chronic hepatitis B," PloS One, vol. 7, no. 3, article e34198, 2012.

[15] A. Kau, J. Vermehren, and C. Sarrazin, "Treatment predictors of a sustained virologic response in hepatitis B and C," Journal of Hepatology, vol. 49, no. 4, pp. 634-651, 2008.

[16] J. P. Shi, J. G. Fan, R. Wu et al., "Prevalence and risk factors of hepatic steatosis and its impact on liver injury in Chinese patients with chronic hepatitis B infection," Journal of Gastroenterology and Hepatology, vol. 23, no. 9, pp. 1419-1425, 2008.

[17] T. Kawaguchi, Y. Sumida, A. Umemura et al., "Genetic polymorphisms of the human PNPLA3 gene are strongly associated with severity of non-alcoholic fatty liver disease in Japanese," PloS One, vol. 7, no. 6, article e38322, 2012.

[18] S. S. Lee, Y. S. Byoun, S. H. Jeong et al., "Role of the PNPLA3 I148M polymorphism in nonalcoholic fatty liver disease and fibrosis in Korea," Digestive Diseases and Sciences, vol. 59, no. 12, pp. 2967-2974, 2014.

[19] M. O. Baclig, J. P. Lozano-Kühne, C. A. Mapua, J. GopezCervantes, F. F. Natividad, and St Luke's Liver Diseases Study Group, "Genetic variation I148M in patatin-like phospholipase 3 gene and risk of non-alcoholic fatty liver disease among Filipinos," International Journal of Clinical and Experimental Medicine, vol. 7, no. 8, pp. 2129-2136, 2014.

[20] S. P. Bhatt, P. Nigam, A. Misra, R. Guleria, R. M. Pandey, and M. A. Pasha, "Genetic variation in the patatin-like phospholipase domain-containing protein-3 (PNPLA-3) gene in Asian Indians with nonalcoholic fatty liver disease," Metabolic Syndrome and Related Disorders, vol. 11, no. 5, pp. 329335, 2013.

[21] E. E. Islek, A. Sazci, M. D. Ozel, and C. Aygun, "Genetic variants in the PNPLA3 gene are associated with nonalcoholic steatohepatitis," Genetic Testing and Molecular Biomarkers, vol. 18, no. 7, pp. 489-496, 2014.

[22] A. Verrijken, S. Beckers, S. Francque et al., "A gene variant of PNPLA3, but not of APOC3, is associated with histological parameters of NAFLD in an obese population," Obesity (Silver Spring), vol. 21, no. 10, pp. 2138-2145, 2013.

[23] Q. Li, H. Q. Qu, A. R. Rentfro et al., "PNPLA3 polymorphisms and liver aminotransferase levels in a Mexican American population," Clinical and Investigative Medicine, vol. 35, no. 4, pp. E237-E245, 2012.

[24] X. E. Peng, Y. L. Wu, S. W. Lin, Q. Q. Lu, Z. J. Hu, and X. Lin, "Genetic variants in PNPLA3 and risk of non-alcoholic fatty liver disease in a Han Chinese population," PloS One, vol. 7, no. 11, article e50256, 2012.

[25] Y. Li, C. Xing, Z. Tian, and H. C. Ku, "Genetic variant I148M in PNPLA3 is associated with the ultrasonography-determined steatosis degree in a Chinese population," BMC Medical Genetics, vol. 13, no. 13, p. 113, 2012.

[26] I. A. Stojkovic, U. Ericson, G. Rukh, M. Riddestråle, S. Romeo, and M. Orho-Melander, "The PNPLA3 Ile148Met interacts with overweight and dietary intakes on fasting triglyceride levels," Genes \& Nutrition, vol. 9, no. 2, p. 388, 2014.

[27] N. T. Krarup, N. Grarup, K. Banasik et al., "The PNPLA3 rs738409 G-allele associates with reduced fasting serum triglyceride and serum cholesterol in Danes with impaired glucose regulation," PloS One, vol. 7, no. 7, article e40376, 2012.

[28] J. H. Park, B. Cho, H. Kwon et al., "I148M variant in PNPLA3 reduces central adiposity and metabolic disease risks while increasing nonalcoholic fatty liver disease," Liver International, vol. 35, no. 12, pp. 2537-2546, 2015.

[29] D. R. Matthews, J. P. Hosker, A. S. Rudenski, B. A. Naylor, D. F. Treacher, and R. C. Turner, "Homeostasis model assessment: insulin resistance and beta-cell function from fasting plasma glucose and insulin concentrations in man," Diabetologia, vol. 28, no. 7, pp. 412-419, 1985.

[30] P. Bedossa, "Utility and appropriateness of the fatty liver inhibition of progression (FLIP) algorithm and steatosis, activity, and fibrosis (SAF) score in the evaluation of biopsies of nonalcoholic fatty liver disease," Hepatology, vol. 60, no. 2, pp. 565-575, 2014, 201.

[31] S. Purcell, B. Neale, K. Todd-Brown et al., "PLINK: a tool set for whole-genome association and population-based linkage analyses," American Journal of Human Genetics, vol. 81, no. 3, pp. 559-575, 2007.

[32] Z. Ye, Z. Li, Y. Wang et al., "Common variants at 10p12.31, $10 \mathrm{q} 21.1$ and 13q12.13 are associated with sporadic pituitary adenoma," Nature Genetics, vol. 47, no. 7, pp. 793-797, 2015.

[33] H. C. Masuoka and N. Chalasani, "Nonalcoholic fatty liver disease: an emerging threat to obese and diabetic individuals," Annals of the new York Academy of Sciences, vol. 1281, no. 1281, pp. 106-122, 2013.

[34] T. Schreuder, B. J. Verwer, C. M. van Nieuwkerk, and C. J. Mulder, "Nonalcoholic fatty liver disease: an overview of current insights in pathogenesis, diagnosis and treatment," World Journal of Gastroenterology, vol. 14, no. 16, pp. $2474-$ 2486, 2008.

[35] D. Wiggins and G. F. Gibbons, "The lipolysis/esterification cycle of hepatic triacylglycerol. Its role in the secretion of very-low-density lipoprotein and its response to hormones and sulphonylureas," The Biochemical Journal, vol. 284, Part 2, pp. 457-462, 1992.

[36] V. W. Dolinsky, D. N. Douglas, R. Lehner, and D. E. Vance, "Regulation of the enzymes of hepatic microsomal triacylglycerol lipolysis and re-esterification by the glucocorticoid 
dexamethasone," The Biochemical Journal, vol. 378, Part 3, pp. 967-974, 2004.

[37] P. A. Wilson, S. D. Gardner, N. M. Lambie, S. A. Commans, and D. J. Crowther, "Characterization of the human patatinlike phospholipase family," Journal of Lipid Research, vol. 47, no. 9, pp. 1940-1949, 2006.

[38] A. C. Lake, Y. Sun, J. L. Li et al., "Expression, regulation, and triglyceride hydrolase activity of adiponutrin family members," Journal of Lipid Research, vol. 46, no. 11, pp. 24772487, 2005.

[39] Y. Huang, J. C. Cohen, and H. H. Hobbs, "Expression and characterization of a PNPLA3 protein isoform (I148M) associated with nonalcoholic fatty liver disease," The Journal of Biological Chemistry, vol. 286, no. 43, pp. 37085-37089, 2011.

[40] M. Kumari, G. Schoiswohl, C. Chitraju et al., “Adiponutrin functions as a nutritionally regulated lysophosphatidic acid acyltransferase," Cell Metabolism, vol. 15, no. 5, pp. 691702, 2012.

[41] E. Smagris, S. BasuRay, J. Li et al., "Pnpla3I148M knockin mice accumulate PNPLA3 on lipid droplets and develop hepatic steatosis," Hepatology, vol. 61, no. 1, pp. 108118, 2015.

[42] M. B. Gandhe and A. R. Srinivasan, "Evaluation of body mass index (BMI) percentile cut-off levels with reference to insulin resistance: a comparative study on south Indian obese and non-obese adolescents," Journal of Clinical and Diagnostic Research, vol. 7, no. 8, pp. 1579-1582, 2013.

[43] C. Kim, B. Kim, N. Joo et al., "Determination of the BMI threshold that predicts cardiovascular risk and insulin resistance in late childhood," Diabetes Research and Clinical Practice, vol. 88, no. 3, pp. 307-313, 2010.

[44] S. Komindr, D. Viroonudomphol, and K. Cherdchu, "Variation of fasting plasma glucose, insulin, and insulin resistance in Thai adults according to the new BMI criteria for Asians," International Journal for Vitamin and Nutrition Research, vol. 78, no. 2, pp. 57-63, 2008.

[45] Y. Miyazaki and R. A. DeFronzo, "Visceral fat dominant distribution in male type 2 diabetic patients is closely related to hepatic insulin resistance, irrespective of body type," Cardiovascular Diabetology, vol. 8, p. 44, 2009.

[46] M. J. Armstrong, J. M. Hazlehurst, D. Hull et al., “Abdominal subcutaneous adipose tissue insulin resistance and lipolysis in patients with non-alcoholic steatohepatitis," Diabetes, Obesity \& Metabolism, vol. 16, no. 7, pp. 651-660, 2014.

[47] D. A. Dumesic, A. L. Akopians, V. K. Madrigal et al., "Hyperandrogenism accompanies increased intra-abdominal fat storage in normal weight polycystic ovary syndrome women," The Journal of Clinical Endocrinology and Metabolism, vol. 101, no. 11, pp. 4178-4188, 2016.

[48] G. Boden, "Role of fatty acids in the pathogenesis of insulin resistance and NIDDM," Diabetes, vol. 46, no. 1, pp. 3-10, 1997.

[49] G. S. Hotamisligil, N. S. Shargill, and B. M. Spiegelman, "Adipose expression of tumor necrosis factor-alpha: direct role in obesity-linked insulin resistance," Science, vol. 259, no. 5091, pp. 87-91, 1993.

[50] P. Sartipy and D. J. Loskutoff, "Monocyte chemoattractant protein 1 in obesity and insulin resistance," Proceedings of the National Academy of Sciences of the United States of America, vol. 100, no. 12, pp. 7265-7270, 2003.
[51] V. Rotter, I. Nagaev, and U. Smith, "Interleukin-6 (IL-6) induces insulin resistance in 3T3-L1 adipocytes and is, like IL-8 and tumor necrosis factor-alpha, overexpressed in human fat cells from insulin-resistant subjects," The Journal of Biological Chemistry, vol. 278, no. 46, pp. 45777-45784, 2003.

[52] U. Smith and B. B. Kahn, "Adipose tissue regulates insulin sensitivity: role of adipogenesis, de novo lipogenesis and novel lipids," Journal of Internal Medicine, vol. 280, no. 5, pp. 465-475, 2016.

[53] F. Tosi, D. Di Sarra, J. M. Kaufman et al., "Total body fat and central fat mass independently predict insulin resistance but not hyperandrogenemia in women with polycystic ovary syndrome," The Journal of Clinical Endocrinology and Metabolism, vol. 100, no. 2, pp. 661-669, 2015.

[54] A. Kempinska-Podhorodecka, M. Krawczyk, M. Klak et al., "Healthy PNPLA3 risk allele carriers present with unexpected body fat composition. A study of one thousand subjects," Journal of Gastrointestinal and Liver Diseases, vol. 23, no. 1, pp. 33-37, 2014.

[55] P. T. Liu, A. C. Hwang, and J. D. Chen, "Combined effects of hepatitis B virus infection and elevated alanine aminotransferase levels on dyslipidemia," Metabolism, vol. 62, no. 2, pp. 220-225, 2013.

[56] S. Petta, C. Cammà, V. Di Marco et al., "Hepatic steatosis and insulin resistance are associated with severe fibrosis in patients with chronic hepatitis caused by HBV or HCV infection," Liver International, vol. 31, no. 4, pp. 507-515, 2011.

[57] Q. Pan, R. N. Zhang, Y. Q. Wang et al., "Linked PNPLA3 polymorphisms confer susceptibility to nonalcoholic steatohepatitis and decreased viral load in chronic hepatitis B," World Journal of Gastroenterology, vol. 21, no. 28, pp. 8605-8614, 2015. 


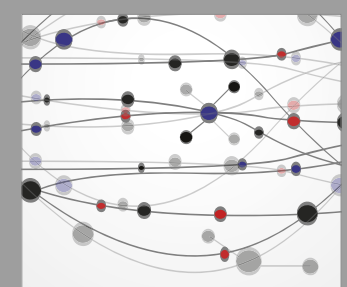

The Scientific World Journal
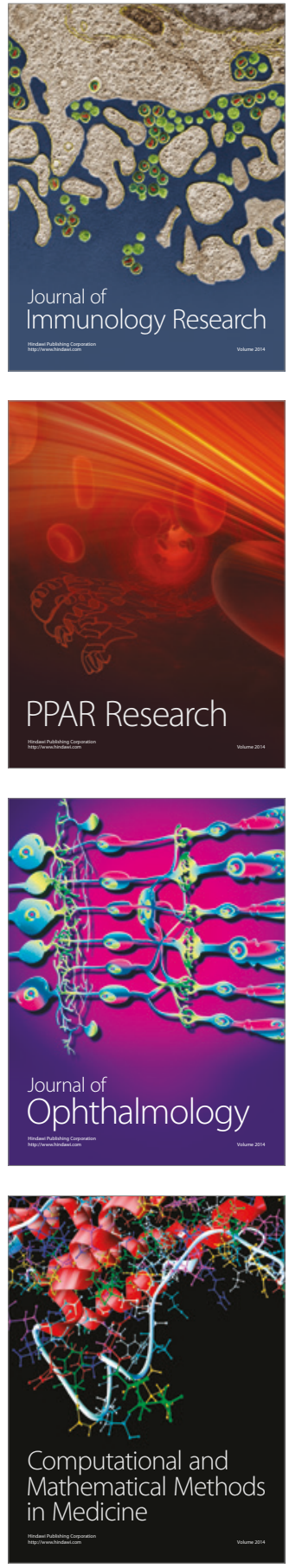

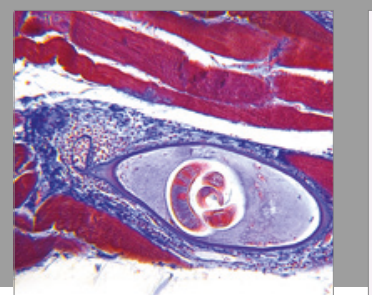

Gastroenterology Research and Practice
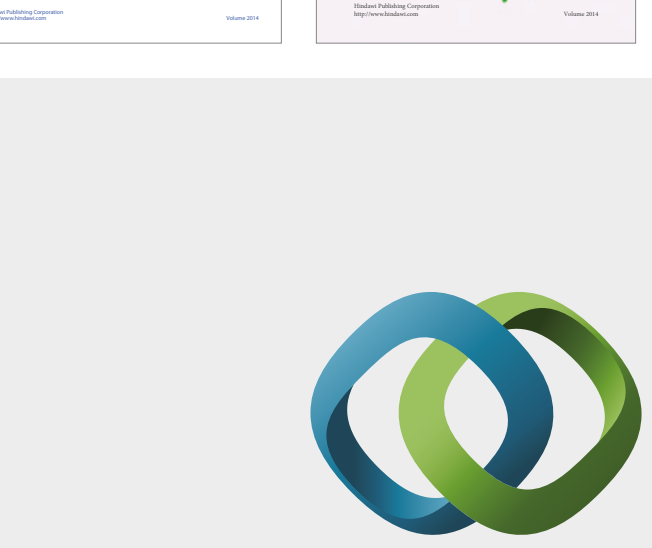

\section{Hindawi}

Submit your manuscripts at

https://www.hindawi.com
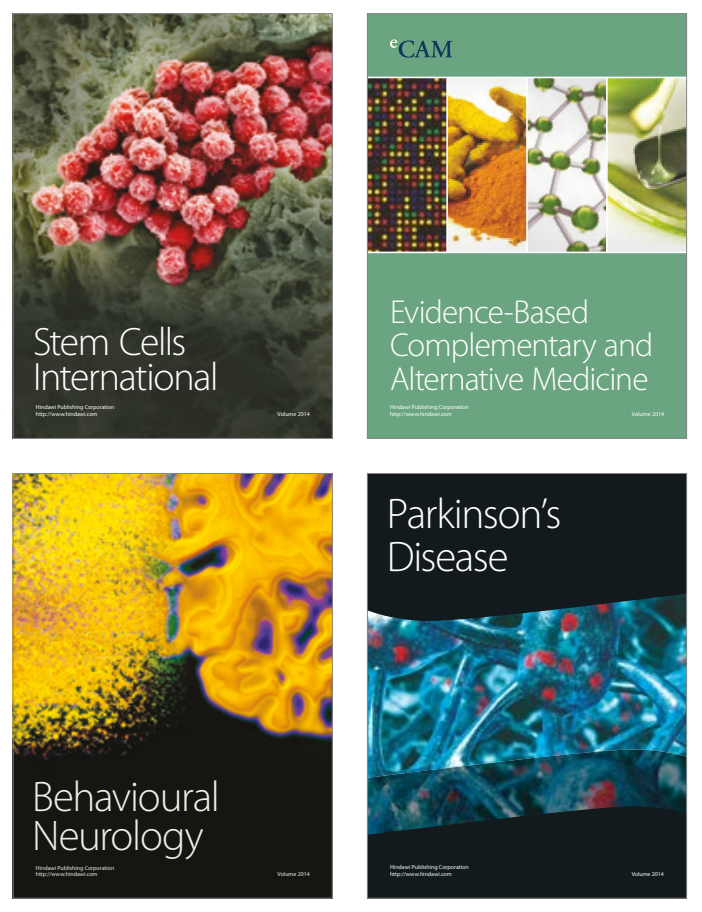
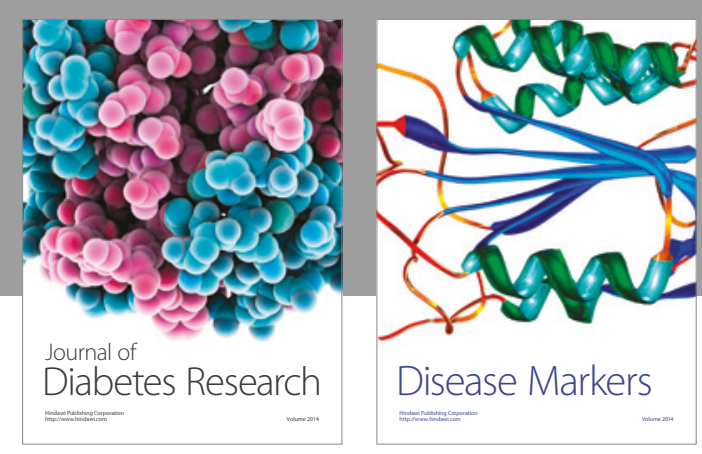

Disease Markers
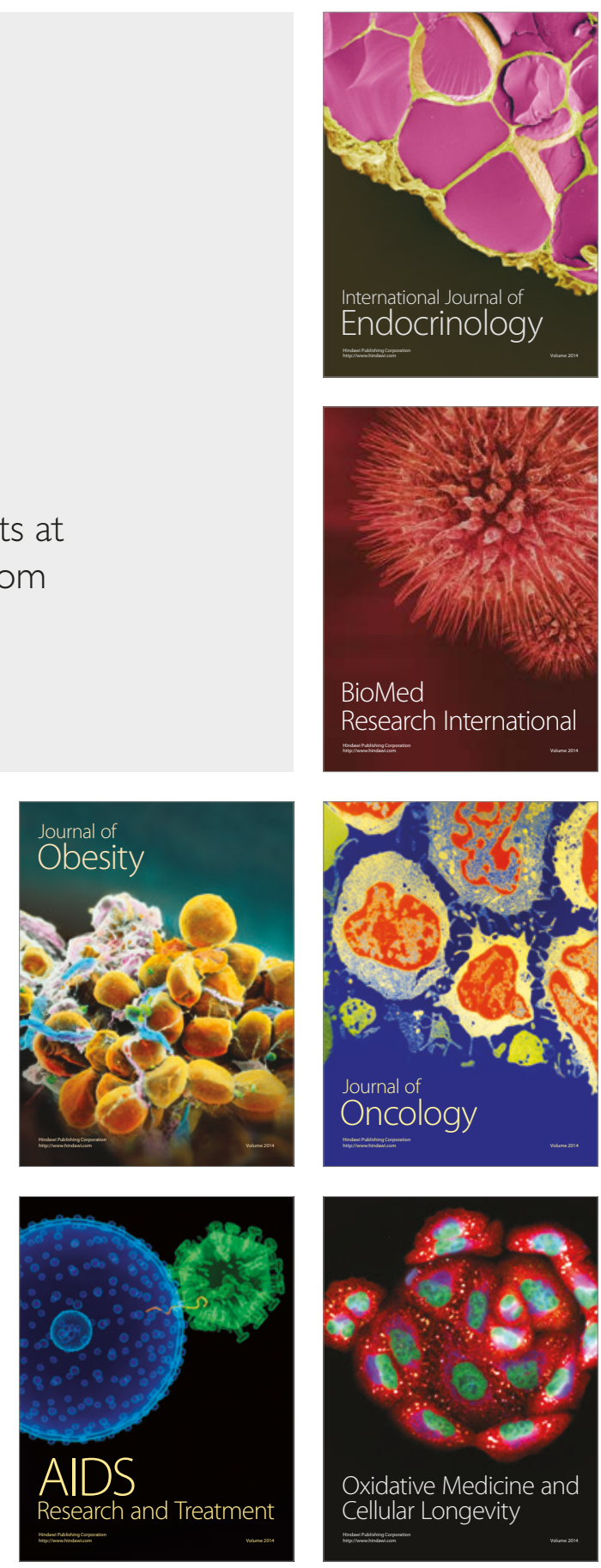